\title{
A Visibility Graph Approach to CNY Exchange Rate Networks and Characteristic Analysis
}

\author{
Can-Zhong Yao ${ }^{1,2}$ and Ji-Nan Lin $^{3}$ \\ ${ }^{1}$ School of Economics and Commerce, South China University of Technology, Guangzhou 510006, China \\ ${ }^{2}$ Physics Department, University of Fribourg, Chemin du Musée 3, 1700 Fribourg, Switzerland \\ ${ }^{3}$ Department of Economics, The Chinese University of Hong Kong, Shatin, Hong Kong \\ Correspondence should be addressed to Can-Zhong Yao; ycz20120911@gmail.com
}

Received 15 April 2017; Revised 19 August 2017; Accepted 24 October 2017; Published 29 November 2017

Academic Editor: J. R. Torregrosa

Copyright (C) 2017 Can-Zhong Yao and Ji-Nan Lin. This is an open access article distributed under the Creative Commons Attribution License, which permits unrestricted use, distribution, and reproduction in any medium, provided the original work is properly cited.

\begin{abstract}
We find that exchange rate networks are significantly similar from the perspective of topological structure, though with relatively great differences in fluctuation characteristics from perspective of exchange rate time series. First, we transform central parity rate time series of US dollar, Euro, Yen, and Sterling against CNY into exchange rate networks with visibility graph algorithm and find consistent topological characteristics in four exchange rate networks, with their average path lengths 5 and average clustering coefficients 0.7. Further, we reveal that all four transformed exchange rate networks show hierarchical structure and small-world and scale-free properties, with their hierarchy indexes 0.5 and power exponents 1.5. Both of the US dollar network and Sterling network exhibit assortative mixing features, while the Euro network and Yen network exhibit disassortative mixing features. Finally, we research community structure of exchange rate networks and uncover the fact that the communities are actually composed by large amounts of continuous time point fractions and small amounts of discrete time point fractions. In this way, we can observe that the spread of time series values corresponding to nodes inside communities is significantly lower than the spread of those values corresponding to nodes of the whole networks.
\end{abstract}

\section{Introduction}

With the enhancement of economy globalization and specialization, international flows of goods, services, capitals, technology, and talents have been connected as dense and intensive networks. Whether the exchange rate of a kind of currency can respond to its real value is significant when we consider exchange rate as the price of money with function of effective allocation for scarce resources in international markets. In 2013, China has become the largest goods trade country with a total amount of 4.16 trillion dollars for goods export and import. In this situation, studies of CNY (Chinese Yuan) exchange rate have attracted attention from international finance, foreign trade, industry development, and macroeconomics prediction.

Since Watts and Strogatz [1] and Barabási and Albert [2] published papers on Nature in 1998 and on Science in 1999, respectively, and revealed small-world and scale-free properties of networks, complex networks theory and its implication have boomed in various studies and make a great influence on research. And they are soon absorbed into physical sciences like system science and social sciences such as sociology, management, and finance as well as economics and make fruitful progress including supply chain management [3], logistics operation [4], commercial networks [5], international trade networks [6], and stocks networks [7]. In the fields of social science, Guo [3] researched on degree distributions of nodes on supply chain networks. Wang and Guo [4] studied the scaling characteristics for aerial inbound logistics operation and proposed a third human dynamics mechanism beyond two classical models proposed by Vazquez et al. [8]. Souma et al. [5] studied topological characteristics of banks and companies networks and further analyzed the influences on organizations and splits of communities caused by merging behaviors. Ren et al. [6] proposed an international trade networks model and analyzed China's position and its 
evolution in networks. Liu and Wang [7] constructed complex networks based on the correlation of stock market prices fluctuation and found the small-world and scale-free properties of stock networks which were similar to those of other complex networks. Consequently, modern networks methods provide a new perspective and gradually become a new instrument for revealing intrinsic regularities and connections in economics system [9] and financial system [10] more comprehensively.

However, there are few studies of exchange rate in a networks perspective so far. Considering exchange rate as a kind of price, some scholars have promoted significant enhancement on studies of price fluctuation with complex networks methods. Yang and Zong [11] researched on price fluctuation network and its topological properties on recycle resources industry of Beijing. Liu et al. [12] studied Shanghai and Shenzhen stock market prices correlation network and its topological properties and analyzed the relationship between fluctuation and liquidity of stocks prices. Y. Yang and $\mathrm{H}$. Yang [13] initially proposed one method of constructing a network of stock time series with correlation matrix, and those works enhanced the basis of networks reconstruction as well as topological analysis of time series. In fields of exchange rate studies, previous studies tended to focus on microfactors and macrofactors which may affect exchange rate fluctuation. Microfactors contain international trade flows, speculation, manipulation of authority, and so forth. Macrofactors include interest rate, inflation, balance of payment, and so forth. In the late 20th century, famous exchange rate models included Mundell-Fleming model, sticky price model by Dornbusch, Flexible Price Monetary Model, Equilibrium Exchange Rate Theory, and Taylor rule proposed by John B. Taylor.

Yang et al. [14] initially utilized visibility graph algorithm to analyze exchange rate time series of US dollar against other international money; meanwhile they discussed the relationship between hierarchical networks structure and Hurst exponents. This work was of great importance in both theory and application. However, worldwide related works on this area are still scarce.

The goal of this paper is to identify the similarity of CNY exchange rate time series of network and to see whether some stable properties exist; therefore, in this way, the conciseness and interpretability of visibility graph model serve a good workable starting point.

The rest of this paper is organized as follows. Section 2 discusses the methodology used in this paper, the data description, and network topological property. Section 3 reveals the hierarchical structured features of the visibility graphs. Section 4 uncovers the small-world and scale-free characteristics of the visibility graph network. Section 5 discusses the node mixing features and Section 6 analyzes the community features of the network. Finally, the concluding remarks are given in Section 7.

\section{Basic Properties Analysis for Exchange Rate Networks Model Based on Visibility Graph Algorithm}

2.1. Model Description. Visibility graph algorithm was a new and fast algorithm for transforming time series into complex networks, proposed by Lacasa et al. [15] in 2008. Owning to some advantages of operability and information reserve, this algorithm was adapted widely to construct complex network from time series transformation. Furthermore, visibility graph algorithm can also be used to verify properties of the original time series, because, even after transformation, the complex networks still reserve as much information of original time series as possible. For example, fractal time series can be transformed into scale-free networks, and, in reverse, the scale-free properties of complex networks can verify the fractal properties of original time series.

The basic idea of visibility graph algorithm can be described as follows: $X=\left\{x_{i}\right\}_{i=1, \ldots, n}$ are time series with $n$ nodes; if any two nodes $\left(t_{a}, x_{a}\right)$ and $\left(t_{b}, x_{b}\right)$ are visible with each other, then $\forall\left(t_{i}, x_{i}\right) \in X$ with $t_{a}<t_{i}<t_{b}$ should satisfy the constraint of

$$
x_{i}<x_{b}+\left(x_{a}-x_{b}\right) \frac{t_{b}-t_{i}}{t_{b}-t_{a}} .
$$

The complex networks after transformation can ensure that each node except the first and the last time point can at least connect with two adjacent ones. And, after transformation, the complex networks are indirect and reserve the visibility after changing the scaling of coordinate axis. The detailed steps to plot the networks can be concluded as follows [15]:

(i) $X=\left\{x_{i}\right\}_{i=1, \ldots, n}$ denotes the time series of exchange rate of CNY against other currencies. Considering $a=1$ and $b=2$, a visible line between the two data points $\left(t_{a}, x_{a}\right)$ and $\left(t_{b}, x_{b}\right)$ can be established if they fulfill (1).

(ii) Set $b=a+1$ and repeat step (i) till $b=n$.

(iii) Set $a=2$; repeat steps (i) and (ii) till $a=n$. We finally get the adjacent matrix.

2.2. Data Description. This paper samples CNY central parity exchange rate time series against US dollar (hereafter USD), Euro, Japanese Yen (Yen), and Great British Pound (Sterling) from the date of financial crisis breaking out September 16, 2008 , to May 8, 2014. The exchange rate networks are, respectively, constructed by visibility graph algorithms: Figures 1(a), $1(\mathrm{c}), 1(\mathrm{e})$, and $1(\mathrm{~g})$ show exchange rate time series fluctuation situation of that time, while Figures 1(b), 1(d), 1(f), and 1(h) show corresponding exchange rate networks transformed by visibility graph of USD, Euro, Yen, and Sterling.

2.3. Network Topological Property. From Table 1, we can find that all indexes of average degree, average clustering coefficient, networks diameter, and path lengths are highly similar on four exchange rate networks, though we can observe relatively large heterogeneous network topology among Figures 1(b), 1(d), 1(f), and 1(h).

It is worth mentioning that the average degree of CNY against USD network is higher than the other three networks. The implication for this situation, regarding the linkage formation rule in visibility graph algorithm, is the appreciation of CNY against USD from August 2010 to May 2014. Moreover, this trend is increasingly going down (i.e., larger 


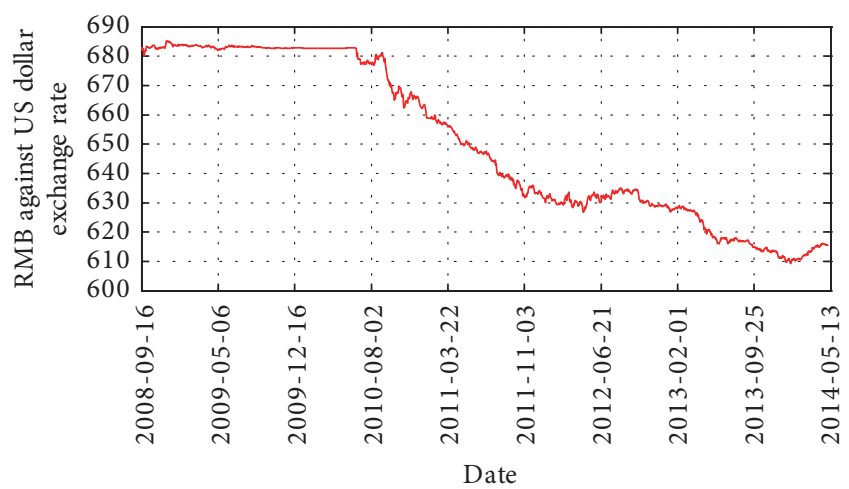

(a) Under direct quotation 100 US dollar against CNY exchange rate time series

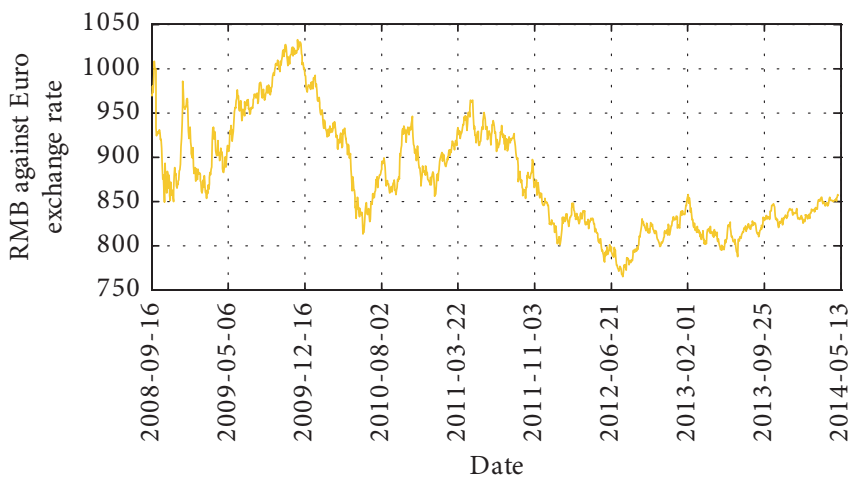

(c) Under direct quotation 100 Euro against CNY exchange rate time series

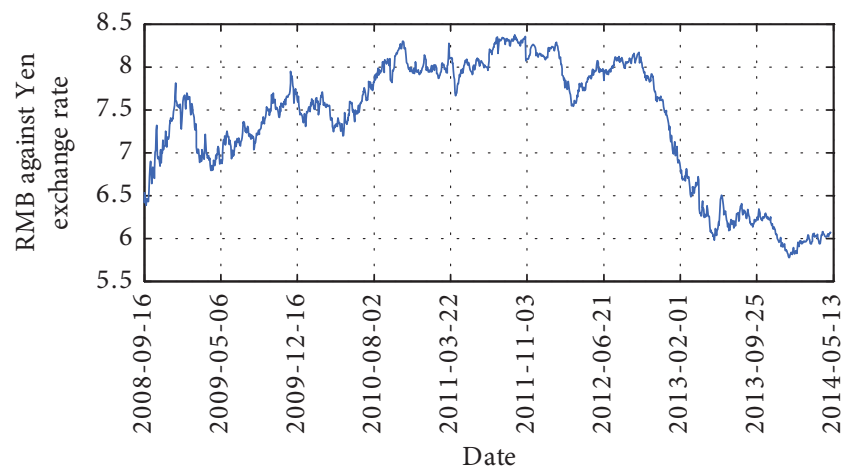

(e) Under direct quotation 100 Yen against CNY exchange rate time series

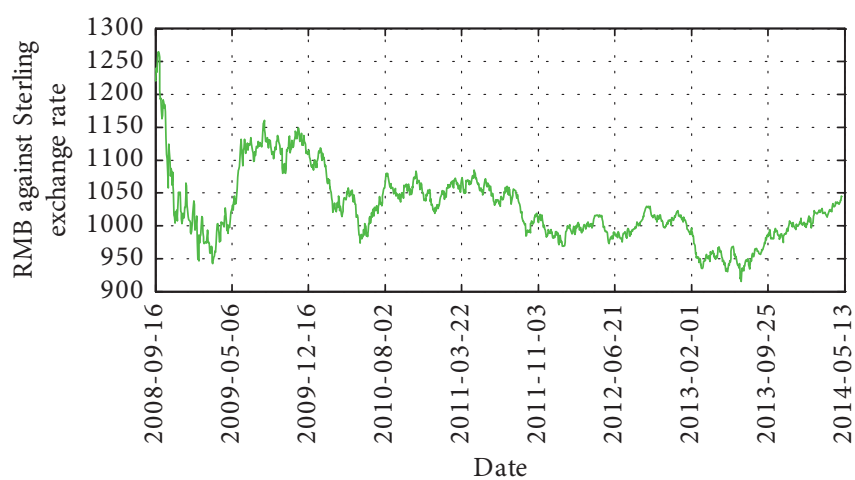

(g) Under direct quotation 100 Sterling against CNY exchange rate time series

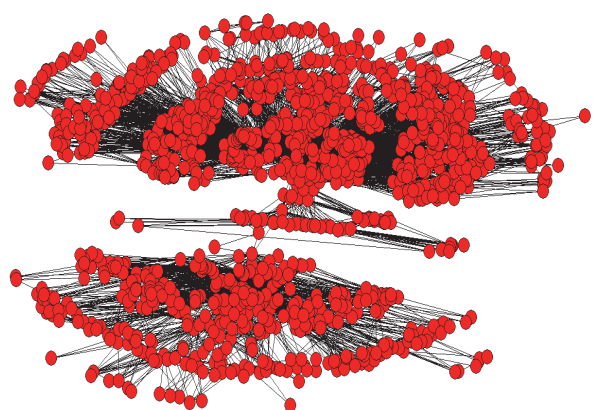

(b) Under direct quotation 100 US dollar against CNY exchange rate networks

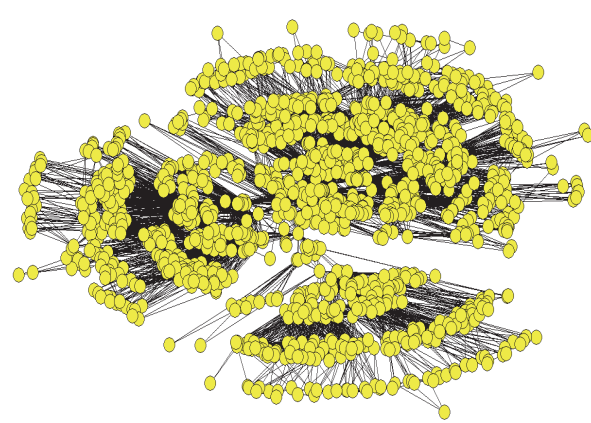

(d) Under direct quotation 100 Euro against CNY exchange rate networks

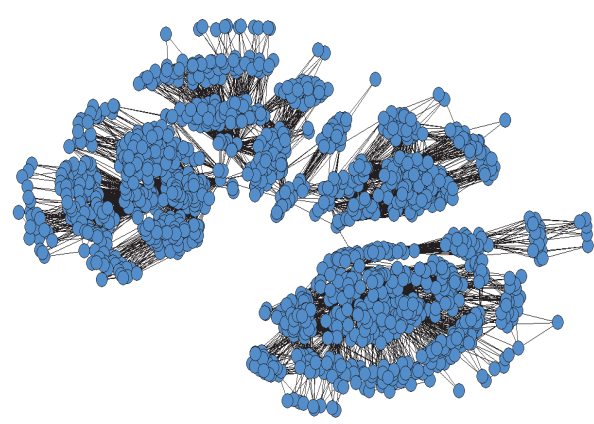

(f) Under direct quotation 100 Yen against CNY exchange rate networks

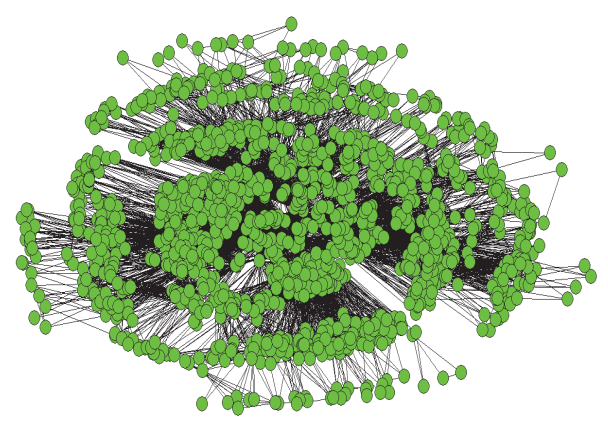

(h) Under direct quotation 100 Sterling against CNY exchange rate networks 
TABLE 1: Basic topological properties of the US dollar, Euro, Yen, and Sterling complex networks.

\begin{tabular}{lccccccccc}
\hline Networks & $N$ & $\langle k\rangle$ & $\langle C\rangle$ & $D$ & $L$ & $\gamma$ & $\beta$ & $\alpha$ \\
US dollar & 1364 & 16.5674 & 0.6785 & 10 & 4.7692 & 1.4503 & 0.7330 & 0.4776 & 0.2509 \\
Euro & 1364 & 13.6100 & 0.7052 & 10 & 4.5664 & 1.4641 & 0.7800 & 0.5519 & -0.2211 \\
Yen & 1364 & 11.9531 & 0.7098 & 11 & 5.6150 & 1.4800 & 0.9103 & 0.5096 & -0.8044 \\
Sterling & 1364 & 14.5601 & 0.6962 & 9 & 4.0800 & 1.4561 & 0.5215 & 0.5486 & 0.0570 \\
\hline
\end{tabular}

Note. $N$ represents network size, $\langle k\rangle$ is average degree of networks, $\langle C\rangle$ stands for average clustering coefficient, $D$ is network diameter, and $L$ is path length. $\gamma$ represents exponent of degree distribution, $\beta$ characterizes small-world property, $\alpha$ stands for hierarchical network, and $r$ measures assortative mixing property.

than the slope between two close time points), and thus a given previous time point in the period can almost link to all time points later without blocks, leading to high average degree values. However, in other networks, peaks and valleys occur alternatively, so the trend cannot last to cause a high average degree value. The average degree, to some extent, can reveal switching pattern of trends within exchange rate time series: a monotone increasing (decreasing) trend or regularly occurring peaks and valleys.

In addition, the smallest value of average clustering coefficient in CNY-USD network can be owed to the almost flat trend from 2008 crisis to August 2010. The authority of foreign exchange of China took advantage of fixed target of CNY against USD. In this case, the condition in the visibility graph was violated greatly in this period for many neighboring time points of given time points, and thus a much lower average clustering coefficient will be achieved during this period regardless of the later period. Therefore, the trend pattern of exchange rate time series will affect the structure of corresponding network through many channels. Given the precise mapping rules, the features of the time series could be explored and investigated from the network perspective.

Tables 2-5 show the time point of hub nodes in different exchange rate networks by measures of centrality and clustering. To determine the centrality, we select three classical local measures to compute the link numbers, connectivity, and clustering for every individual node, respectively, in visibility graphs of CNY exchange rates, and, based on their values, we list up top 10 nodes and the corresponding date in every network. The clustering coefficients in Tables 2-5 are local clustering coefficients for every individual node.

Taking Table 2 as an example, in the CNY-USD exchange rate network, we rank all nodes, from high to low, by their values of degree, betweenness centrality, and clustering coefficient.

As for degree, the highest degree value of all nodes is 165 , and the corresponding date of time series value for this node with highest degree value is September 1, 2010. And, in this network, the node amount is 1 which means we have only one such node with highest degree value. As for betweenness centrality, the highest betweenness centrality value is 0.491 , and the corresponding date of time series value for this node with highest betweenness centrality value is also September 1 , 2010. And, in this network, such node with highest betweenness centrality value is also unique. Regarding clustering coefficient, the maximum value of clustering coefficient is 1; however, such nodes are as many as 344 and one of them has the corresponding date in time series September 18, 2008.
In networks, the larger degree of a node tends to reveal certain heterogeneity of corresponding time series value. Taking relatively fractional Yen exchange rate networks (Figure $1(\mathrm{~g})$ ) as example, the node which serves as the only bridge to connect two communities is in response to the date 2010-1102 in time series (Figure 1(e)), and the value of the date 2010$10-26$ is verified to rank top $11 \%$ degree and second largest betweenness centrality. It means that the continuous time series could be divided into two parts. The whole complex network shows the characteristics of two significant independent communities.

On the contrary, we cannot figure out clearly any node which served as "local maximum value" in the Sterling networks (Figure 1(h)). Compared with Yen's time series, we find that the length of period in Sterling's time series is shorter than that of Yen's, and "local maximum value" of Sterling's time series is not much higher than its neighboring values. As a result, "local maximum value" in Sterling's networks is hard to separate time series completely and evolve into a more collective network.

\section{Hierarchical Structured Analysis for Exchange Rate Networks}

Calculate weighted average value of clustering coefficients for nodes with degree $k$ :

$$
\bar{C}=\langle C \mid k\rangle=\frac{\sum_{i=1}^{n} C_{i}}{n}
$$

where $n$ stands for the amount of different clustering coefficients for nodes with degree $k$. It can be believed that the network follows hierarchical structure if $\bar{C}(k) \sim k^{-\alpha}$.

This paper finds that all exchange rate networks follow relatively consistent hierarchical structures, as shown from Figures 2(a)-2(d), and the calculating results of $\alpha$ are basically equal to 0.5 .

The hierarchical structure coefficients reveal, for lowdegree nodes, that they have highly dense links with their neighboring nodes and lead to relatively higher clustering coefficient; for extremely high-degree nodes with relatively low clustering coefficient, their effects are only to connect different components. We calculate the correlative coefficient between clustering coefficient and degree for CNY against US dollar, Euro, Yen, and Sterling exchange rate networks and find that those correlative coefficients are, respectively, $-0.749,-0.798,-0.802$, and -0.784 , following significantly negative correlative relationship. 


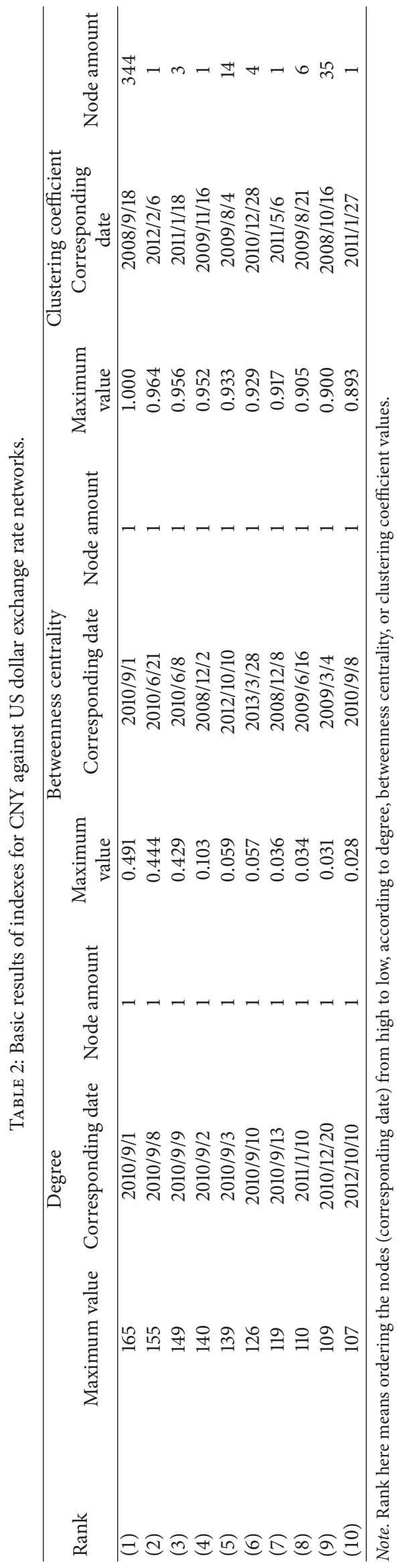




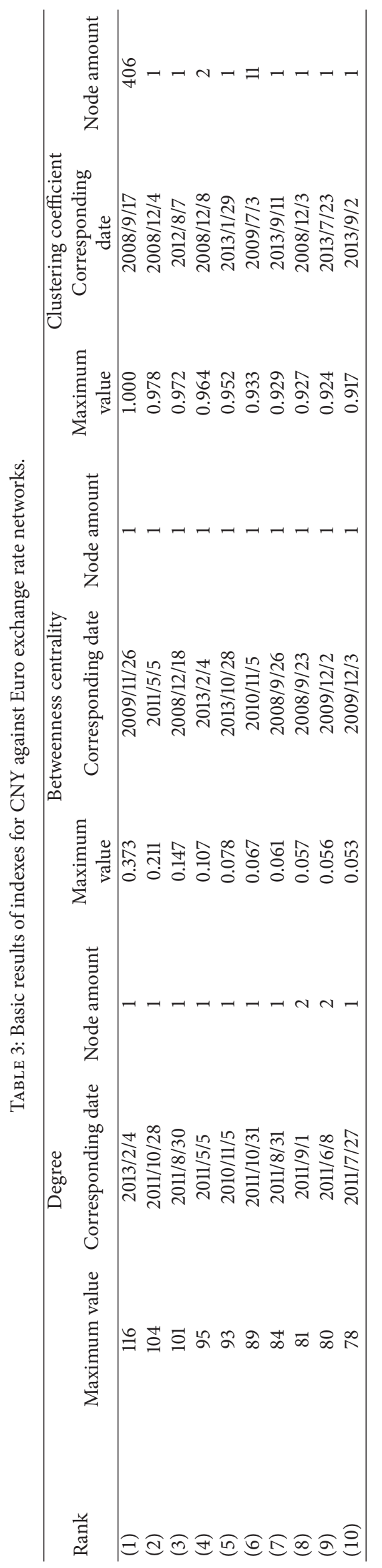




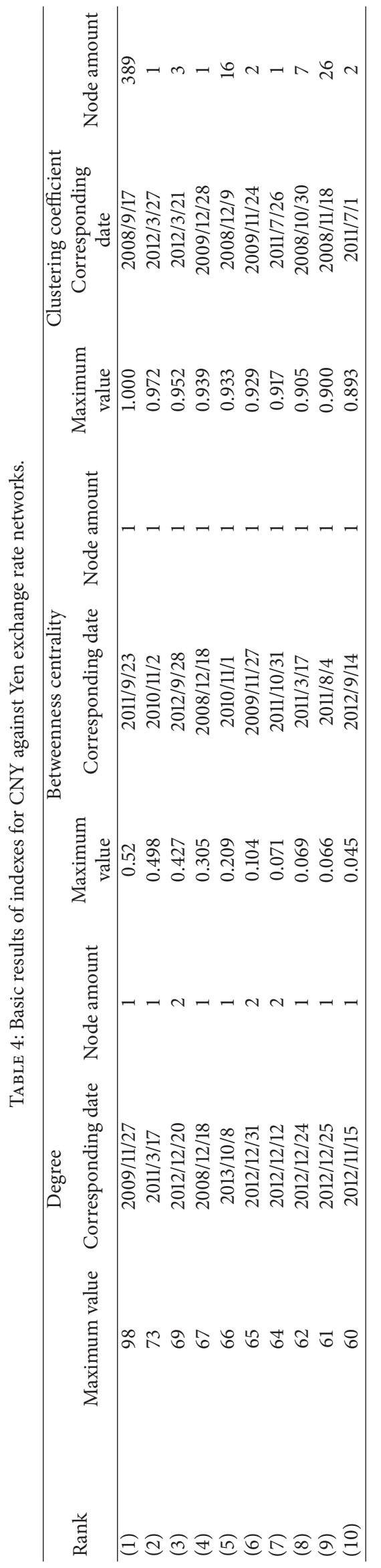




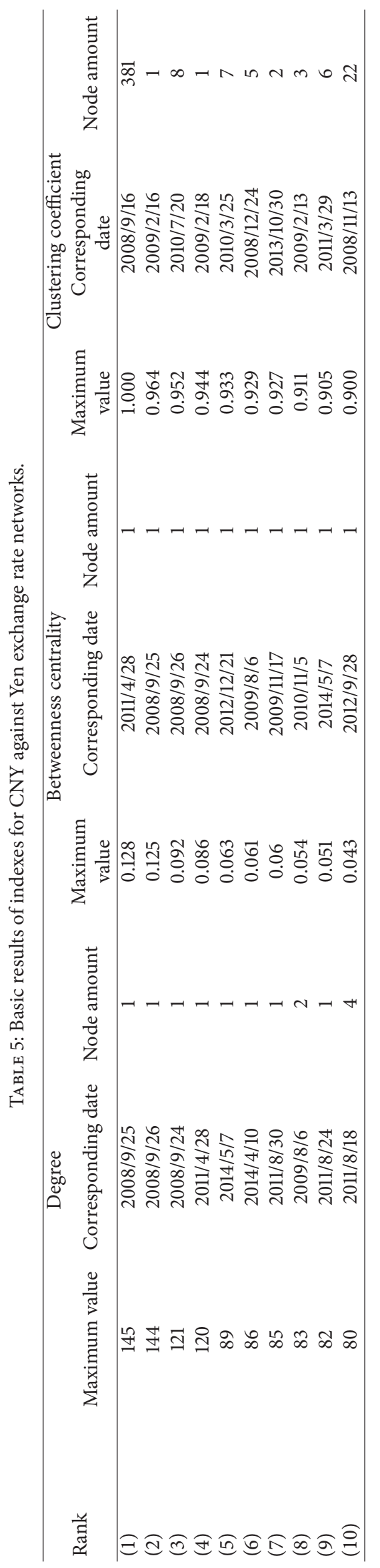




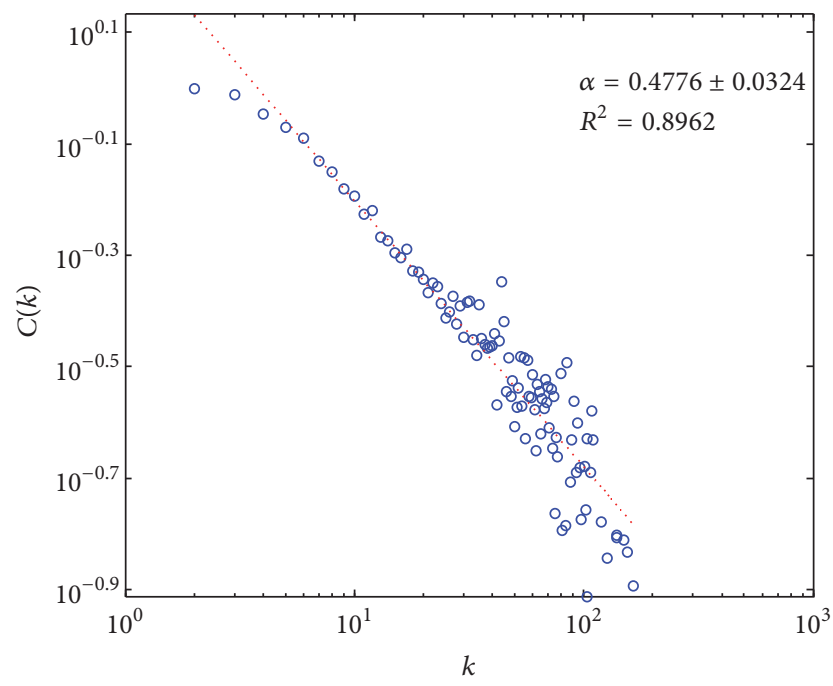

(a) Hierarchical structure of CNY against US dollar exchange rate network

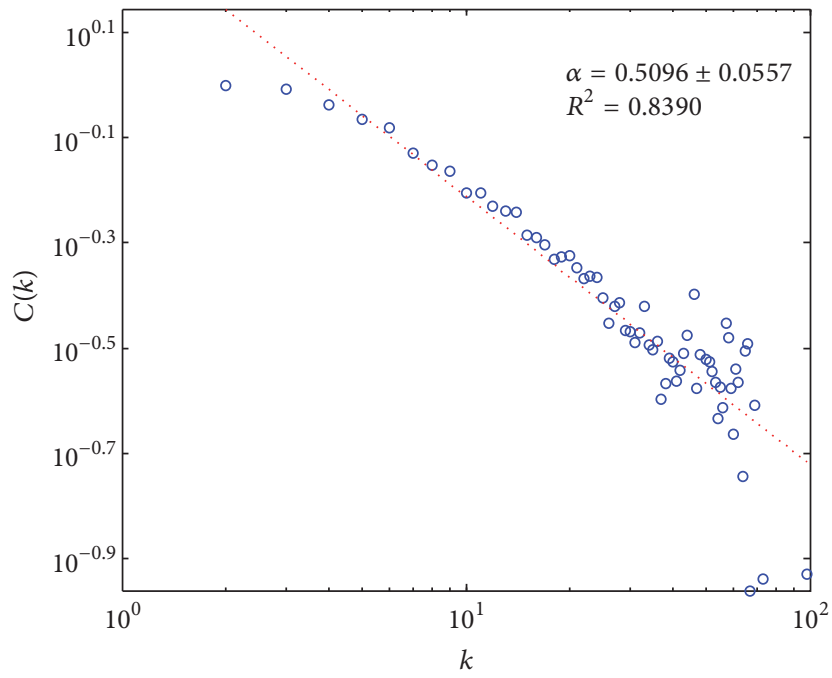

(c) Hierarchical structure of CNY against Yen exchange rate network

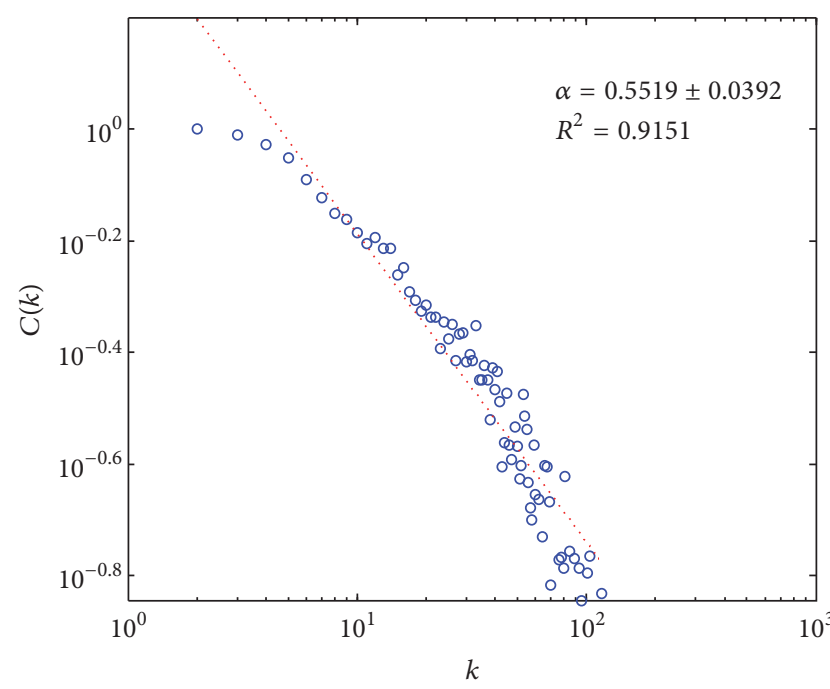

(b) Hierarchical structure of CNY against Euro exchange rate network

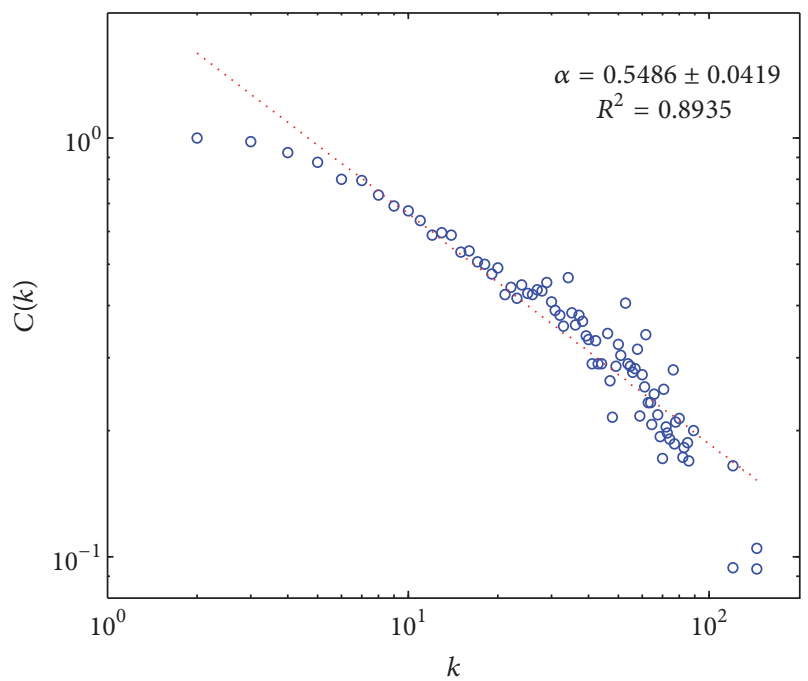

(d) Hierarchical structure of CNY against Sterling exchange rate network

Figure 2

As to a hierarchical structured network, we can propose that there are probably many small-scale groups of nodes: within the group, nodes are densely linked; between the groups, groups are loosely connected. And according to our visibility graph algorithm for networks transformation, we figure out that a high-degree hub node has a higher possibility to link with another higher-degree hub node; however a low-degree node has a low possibility to connect with a higher-degree hub node. The implication of this node linkage pattern can also be referred to the switching pattern of trends during different periods of exchange rate time series. The peak and valley time point tend to have higher probability to form more links, so the recursively occurring peaks and valleys probably generate a class of high-degree points to hierarchically connect with low-degree points between two peaks.

As shown in Figures 1(a), 1(c), 1(e), and 1(g), there exist large differences in various exchange rate sequences; that is, at a certain time point, a higher value of CNY/US exchange rate probably does not tend to correspond to a higher value of $\mathrm{CNY/EURO} \mathrm{exchange} \mathrm{rate.} \mathrm{And} \mathrm{the} \mathrm{consistent} \mathrm{hierarchical}$ structure scaling among various currencies reveals a selforganization property of exchange rate network system. Therefore we propose that large fluctuation in some time points is not an unusual phenomenon but a rule emerging in systemic self-organization process.

\section{Small-World and Scale-Free Property for Exchange Rate Networks}

4.1. Small-World Property. As observing from Figures 3(a)-3(d), we can, respectively, figure out classical smallworld property of networks transformed from CNY against US dollar, Euro, Yen, and Sterling exchange rate networks. Besides, we find that the average clustering coefficients of 


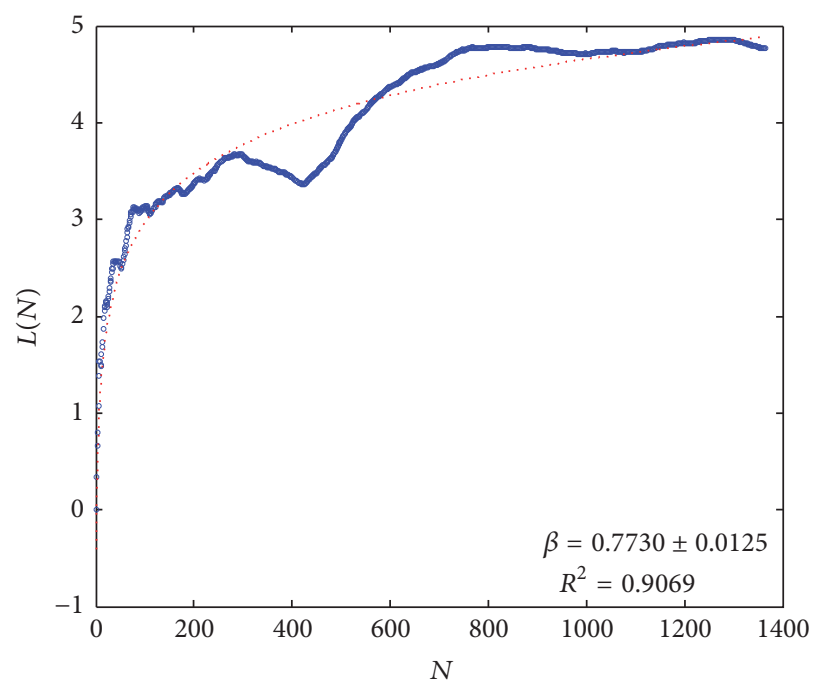

(a) Small-world property of CNY against US dollar exchange rate network

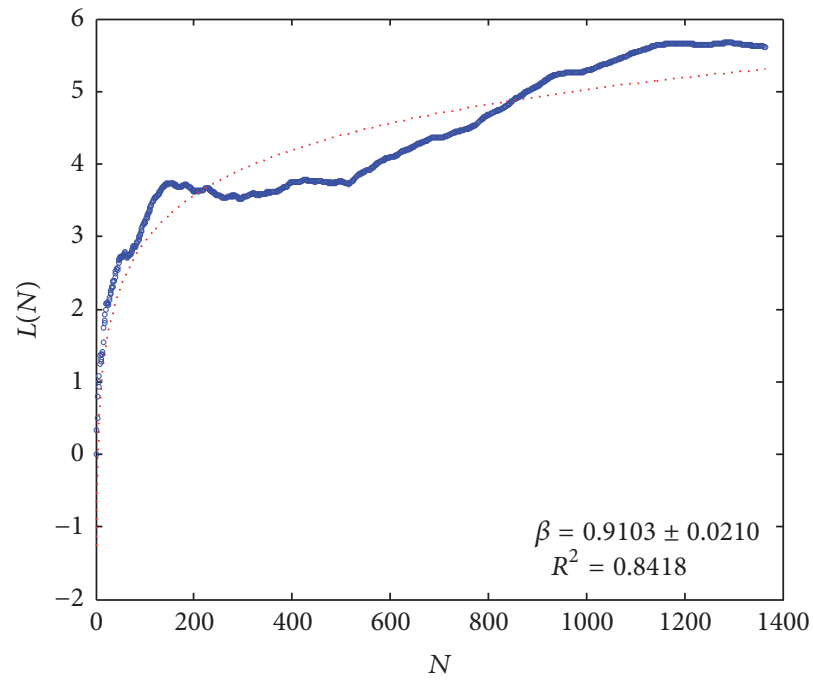

(c) Small-world property of CNY against Yen exchange rate network

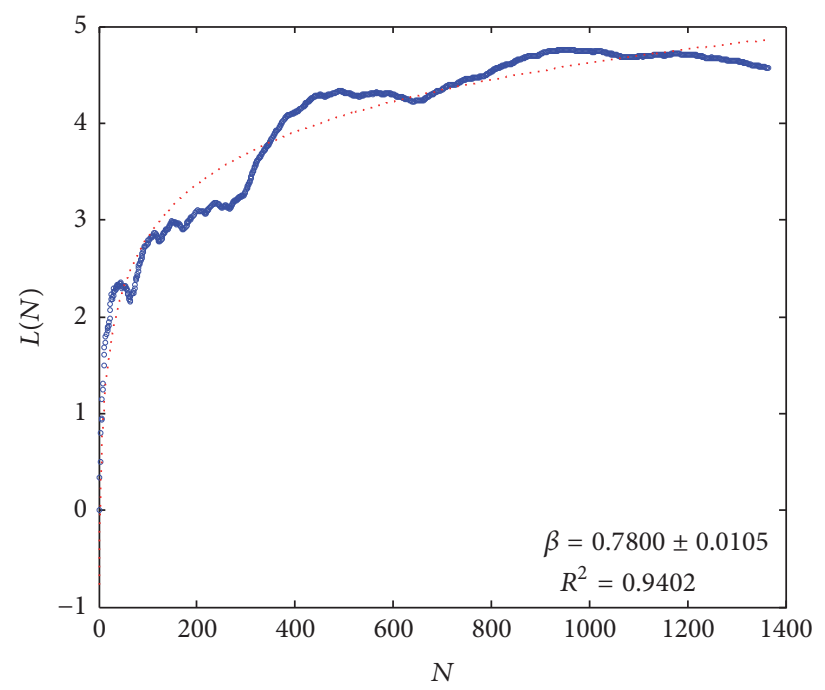

(b) Small-world property of CNY against Euro exchange rate network

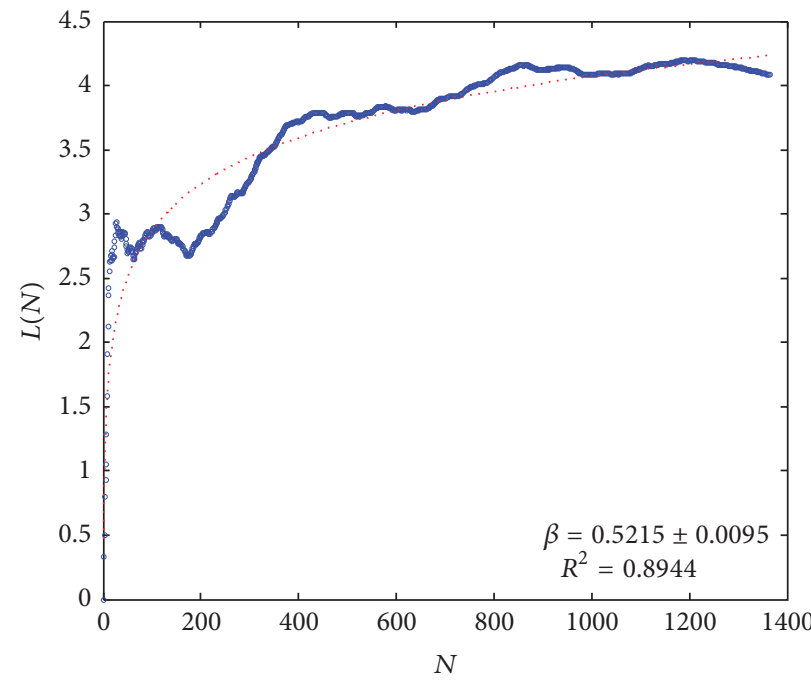

(d) Small-world property of CNY against Sterling exchange rate network

Figure 3

those networks are up to 0.7. And, referring to Table 1, we find that their average path lengths are relatively short, below 5 , except for CNY against Yen exchange rate network, whose value is 5.6.

Average path length of every exchange rate network is 5 , and average clustering coefficient of networks is almost 0.7 , verifying certain intrinsic correlation among exchange rate time series. This property of four exchange rate networks is basically consistent, which cannot be acquired on a singledimensional time series analysis.

4.2. Scale-Free Property. Power-law distribution is also called Pareto distribution, which exists widely in various complex networks with diverse structures and functions. In recent decades, scientists have found power-law distribution, a kind of universal law in characterizing distributions of time intervals, space intervals, and other important statistics in nature and society. And so far scientists have found some mechanisms of power-law generation, such as preferential attachment [2], random process [16], self-organized criticality [17], and highly optimized tolerance [18], and those models can explain partially how power-law does emerge.

With research on many real systems, we find that in large amount of self-organization systems, their behavioral dynamics intervals tend to follow power-law features [1922]. As for exchange rate system of a country, if the force of exchange rate fluctuation was completely from market or closely from market, the interval distribution should follow consistent power-law distribution regardless of any choice of foreign currency as measurement.

According to our visibility graph algorithm, the connections among nodes are decided by visibility principle. So a high-degree node under this network should follow significantly two properties: first, the degree of this node is 


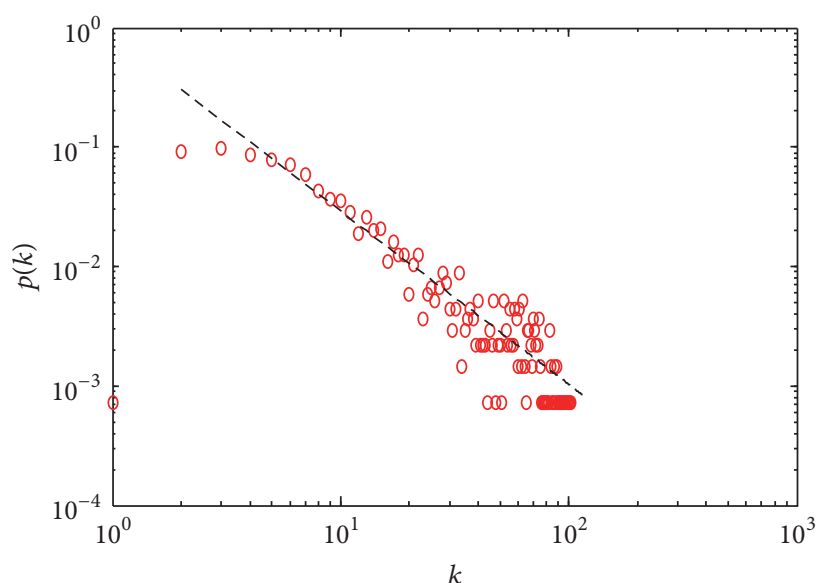

(a) Degree distribution of CNY against US dollar exchange rate network

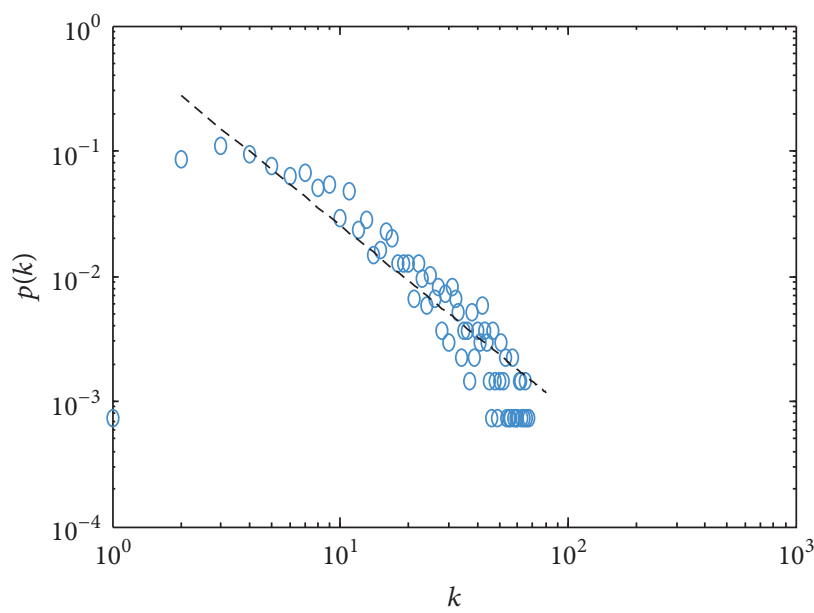

(c) Degree distribution of CNY against Yen exchange rate network

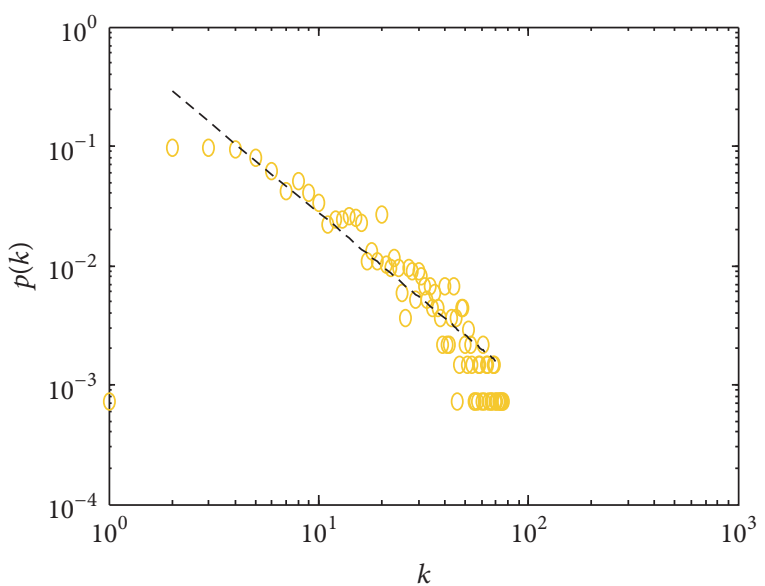

(b) Degree distribution of CNY against Euro exchange rate network

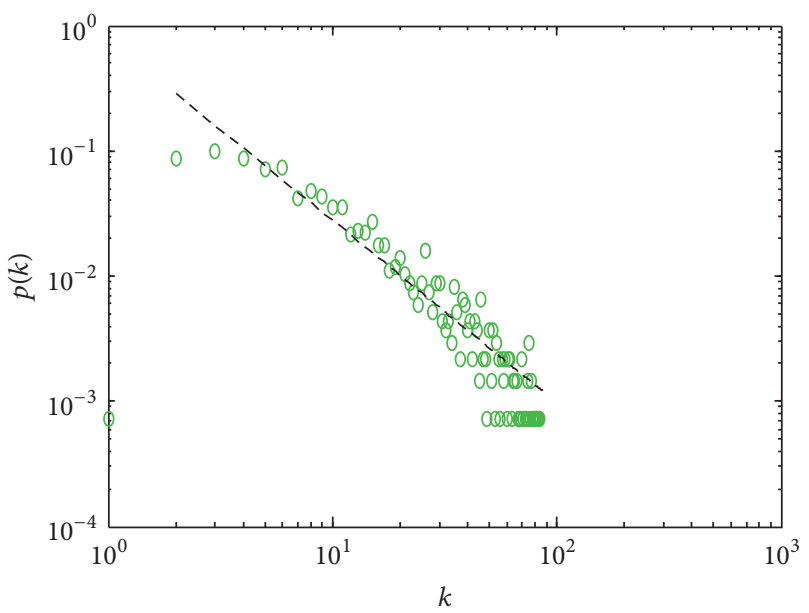

(d) Degree distribution of CNY against Sterling exchange rate network

FIGURE 4

significantly greater than other neighboring nodes; second, as nodes are closer to this node, the degree of those nodes will increase more quickly, and as nodes are farther from this node, the degree of those nodes will decrease more slowly.

We utilize the Maximum Likelihood Estimation proposed by Clauset et al. [23] to fit the distribution and find surprisingly that the power exponents of US dollar exchange rate network, Euro exchange rate network, Yen exchange rate network, and Sterling exchange rate network are approximately 1.5 (Figure 4$)$ ( $\beta$ in Table 1 ).

As shown in Figures 1(a), 1(c), 1(e), and 1(g), although their original time series seem to be different in numerical value, fluctuation range, change trend, and so forth, we witness the consistency in scale-free property with visibility graph algorithm. We can even find in the time from September 19, 2008, to June, 2010, that the values of 100 US dollars against CNY exchange rate time series remain almost steady However, the fluctuations of Euro, Yen, and Sterling exchange rates are significantly different from the US exchange rate at a certain time.

From Tables 2-5, although the dates of large-index-value nodes of networks are of great difference, scaling exponents of every network are almost the same. Therefore, we initially consider the whole exchange market is a self-organization system. Even regulated by the authority to some extent, the exchange rates' systems still follow consistent scaling law.

Combining the regime of CNY exchange rate and visibility graph algorithm, we argue that, first, large shocks in economic system cause power-law tails and maximum values of nodes' degree, such structural turning point of international trade under financial crisis, large volume of buy-back or bear sales by central bank, and an avalanche of hot money flowing in or out, second, the normal demand-supply relationship of real section or capital section of CNY against US dollar, Euro, Yen, and Sterling determines the middle part of degree distribution, and, third, the manipulated floating range of CNY decided by central bank and amounts of arbitrage investors synthetically lead to head of degree distribution. Due to the manipulated floating range, the maximum value of nodes' degree and their increasing relationship cannot be completely satisfied. Therefore, many similar-value points in time series reflect groups of low-degree nodes and cause the head part of power-law distribution. 


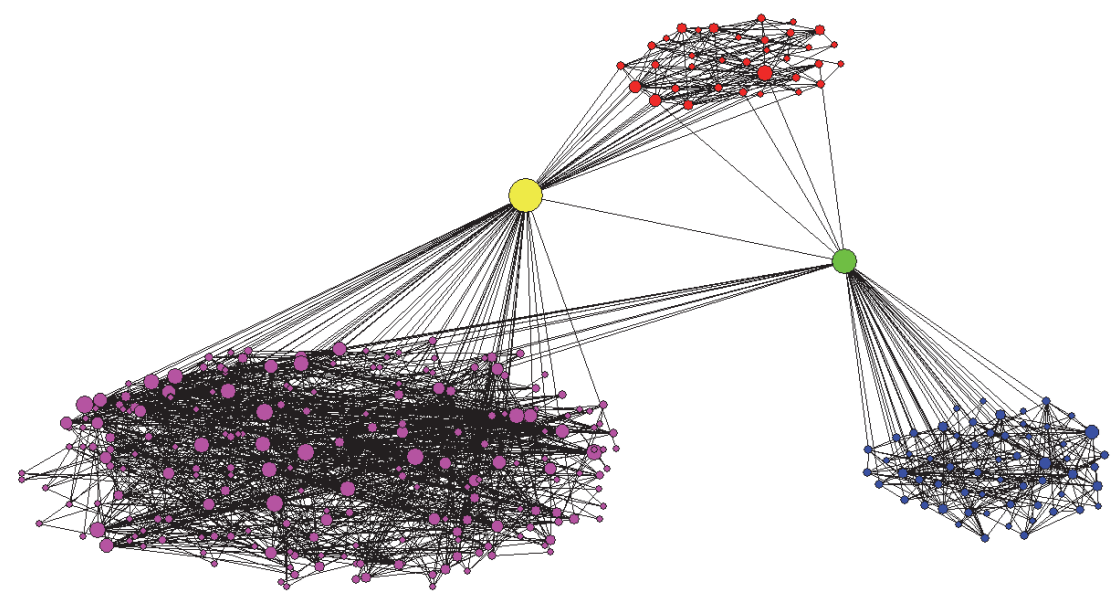

FIGURE 5: Diagram of two interactive forces of node mixing inside and among groups.

The analysis of degree distribution reveals the maximum value of nodes' degree and their dynamics features caused by time series fluctuation from a global perspective. However, the maximum values of time series not only cause a powerlaw distribution but also naturally divide time series into topological network community. This natural separation of time series can hardly be observed from one-dimension analysis of time series, so we go deeply into exploration of their division mechanism.

\section{Node Mixing Features Analysis on Exchange Rate Networks}

We utilize the Pearson correlation coefficient $r(-1 \leq r \leq 1)$ of node degree to characterize the property of node mixing. The definition of $r$ is

$r$

$$
=\frac{M^{-1} \sum_{i} k_{1} k_{2}-\left[M^{-1} \sum_{i}(1 / 2)\left(k_{1}+k_{2}\right)\right]^{2}}{M^{-1} \sum_{i}(1 / 2)\left(k_{1}^{2}+k_{2}^{2}\right)-\left[M^{-1} \sum_{i}(1 / 2)\left(k_{1}+k_{2}\right)\right]^{2}},
$$

where $k_{1}$ and $k_{2}$, respectively, stand for the degree of two ends for edge $i$; $M$ represents the total amount of edges in network. If $r>0$, the network exhibits assortative mixing; if $r<0$, the network exhibits disassortative mixing; if $r=0$, the network is not correlative.

Our results show that both US dollar exchange rate network and Sterling exchange rate network exhibit assortative mixing, and both Euro exchange rate network and Yen exchange rate network exhibit disassortative mixing. In addition, the extent of disassortative mixing for Yen exchange rate network is as high as up to -0.8044 .

We propose that the node mixing property reveals the relationship between node-level interactive force among nodes and group-level interactive force among groups (Figure 5): first, there are interactions of nodes within a given group, and due to the similarity of nodes' degree value within a group, this first kind of force is positive toward wholenetwork node mixing property; second, the hierarchical structure causes a force of a high-degree node linking with a group of lower-degree nodes, which is negative toward whole-network node mixing property. Whether the wholenetwork node mixing is assortative or disassortative mainly depends on the dominance of first force against the second force (Figure 5).

Considering the fluctuation features of CNY against US dollar, Euro, Yen, and Sterling exchange rate time series, we can observe that the amount of maximum value and hierarchy strength of both US dollar and Sterling are lower than those of Euro and Yen. This is because both US dollar and Sterling have a smaller fluctuation and shorter cycles in time series, reflecting negative interactive forces and assortative mixing pattern in network. Besides, the fluctuation of Sterling is still greater than that of US dollar, so the assortative mixing pattern of Sterling tends to be weaker than that of US dollar. Both Euro and Yen are greater in fluctuation in time series, revealing that, in Euro and Yen exchange rate network, the negative interactive forces among groups are significantly greater than their positive interactive force within group and emerge into a disassortative mixing pattern. This conclusion is consistent with the significant hierarchical structure of Yen exchange rate network (Figure $1(\mathrm{f})$ ).

\section{Community Analysis of Exchange Rate Network}

6.1. Review of Community Algorithm. Community structure reflects the heterogeneity on connection distribution within network. A community tends to consist of similar-function or consistent-property nodes, and hence community analysis is considered of great importance in revealing the relationship between structure and function. In 2002, Girvan and Newman [24] initially proposed community structure of social network and biology network on PNAS and attracted attention from scholars of complex networks. Besides, Newman and Girvan [25] also provided an index called modularity 
for measuring community division goodness. The core of modularity is to compare divided-community structure with structure in random network. The definition of modularity is

$$
Q=\frac{1}{2 m} \sum_{i j}\left(A_{i j}-\frac{k_{i} k_{j}}{2 m}\right) \cdot \delta\left(C_{i}, C_{j}\right),
$$

where $k_{i}$ and $k_{j}$ are degree of a node; $C_{i}$ is the community to which node $i$ belongs; $m$ is the total amount of edges; when $C_{i}=C_{j}, \delta\left(C_{i}, C_{j}\right)=1$; otherwise, $\delta\left(C_{i}, C_{j}\right)=0$. The value of $Q$ tends to be within the range between 0 and 1 , and, generally speaking, $Q=0.3$ is the lower bound for significant community structure of a network.

As observed from Figures 1(b), 1(d), 1(f), and 1(h), we can guess the significant community structure of exchange rate networks transformed from CNY against US dollar, Euro, Yen, and Sterling exchange rate time series; that is, nodes inside a group are densely connected; however, among groups, the connections are relatively sparse. We can also figure out that in Figure 1(b) the CNY against US dollar exchange rate networks can be approximately divided into 2 communities, in Figure 1(d) the CNY against Euro exchange networks can be roughly separated into 3 communities, in Figure $1(\mathrm{f})$ the $\mathrm{CNY}$ against Yen exchange rate network can be divided into 2 communities, and in Figure 1(h) the CNY against Sterling exchange rate network can be separated roughly into 2 communities.

Due to large computational complexity, limit distinguishability, and single scaling of traditional GN algorithm, we take advantage of Louvain algorithm to detect community structure of US dollar, Euro, Yen, and Sterling exchange rate networks, respectively. Louvain algorithm [26] is also based on modularity and traverse. Its main idea is calculating the marginal increasing modularity after extracting one node from its original community and adding to every other community. And then we move this node into that community with largest marginal increasing modularity, repeating those steps until no nodes can be moved for higher modularity. The marginal increasing modularity is

$$
\Delta Q=\frac{2 K_{a, \text { in }}}{2 m}-\frac{2 \sum \text { tot } \times K_{a}}{(2 m)^{2}},
$$

where $K_{a, \text { in }}$ represents the sum of weights of all edges from $i$ to $C ; \sum$ tot stands for the sum of weights of all edges connected to community $C$.

6.2. Application of Louvain Algorithm. The results show significant community structure of CNY against US dollar, Euro, Yen, and Sterling exchange rate networks. The community detection algorithm reveals the results that the nodes are building the dense connections inside the same community and sparse connections among different communities. All modularity indexes of exchange rate networks are above 0.6; further, both modularity indexes of Euro and Yen exchange rate networks are higher than 0.7. The results reflect that we gain a great effect of community detection, which is significantly higher than the lower bound, 0.3 .
Besides, we can also find that the community structured property of Figures 6(a), 6(b), 6(c), and 6(d) is corresponding to our intuitive observation on Figures 1(b), 1(d), 1(f), and 1(h). Because CNY has been fixed with US dollar since the financial crisis breaking out in September 16, 2008, the little fluctuation of CNY against US dollar caused inferior community-detection effect compared with the other 3 networks.

Power-law distribution is helpful to explain the amount of maximum value on CNY exchange rate time series; however, community detection, to some extent, can help to explain the distributed location of those maximum values on $\mathrm{CNY}$ exchange rate time series and the relationship among those maximum values.

Furthermore, we mark nodes of communities according to their order in time series and expand to every network. After summarizing the characteristics of the nodes inside each community and their corresponding time points, we find that the communities could be classified into two categories: first, nodes within the same community are from a sequential part of the time series, and this kind of community tends to be relatively in small scale; second, small percentage of communities consists of several fragments, and this kind of community tends to be in large scale.

In order to explore the property of second kind of community, that is, those communities consisting of several fragments of time series, we choose the largest-size communities of US dollar, Euro, Yen, and Sterling exchange rate network, which are, respectively, US dollar community 6, Euro community 6 , Yen community 11 , and Sterling community 8 . We can easily find that the largest-size community and the community containing the most fragments are mostly consistent except Sterling community. In Sterling community, the second largest-size community 2 has more fragments than the largest-size community 8 . Here we choose Sterling community 2 for further discussion.

The term fragment discussed in Table 6 can be defined as a short sequence of time point with continuous-time record. Let us say, in a CNY exchange rate time series $\left\{x_{i}\right\}_{i=1, \ldots, n}$, the community $k$ only absorbs $\left\{x_{i}\right\}_{i=1, \ldots, m},\left\{x_{i}\right\}_{i=n, \ldots, j, n>m+1}$, and $\left\{x_{i}\right\}_{i=q, \ldots, t, q>j+1}$. Then we will say community $k$ contains 3 fragments of CNY exchange rate time series points.

According to the statistical results, we find that, in community containing the most fragments of US dollar, Euro, Yen, and Sterling exchange rate network, the population variance of time series values inside community containing the most fragments is greatly smaller than that of whole time series. In addition, the maximum value inside community containing the most fragments is extremely close to the maximum value of whole time series.

As shown in Table 7, the nodes inside community tend to correspond to time series which have much smaller population variance of value than whole time series. In addition, those maximum values of community containing the most fragments tend to correspond to nodes with the greatest degree, betweenness centrality, or clustering coefficient and the largest values in time series. For example, the maximum value of US dollar community 6 is also the greatest-degree and greatest-betweenness-centrality node of whole US dollar 


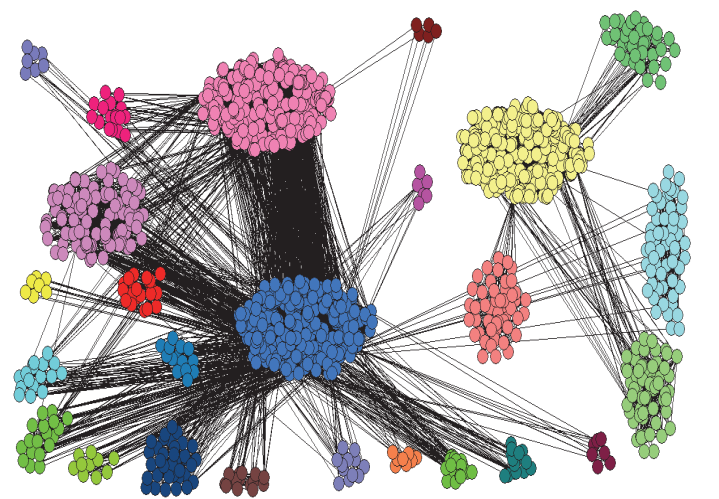

Number of clusters: 25

Modularity: 0.636014

(a) Community detection of CNY against US dollar exchange rate network

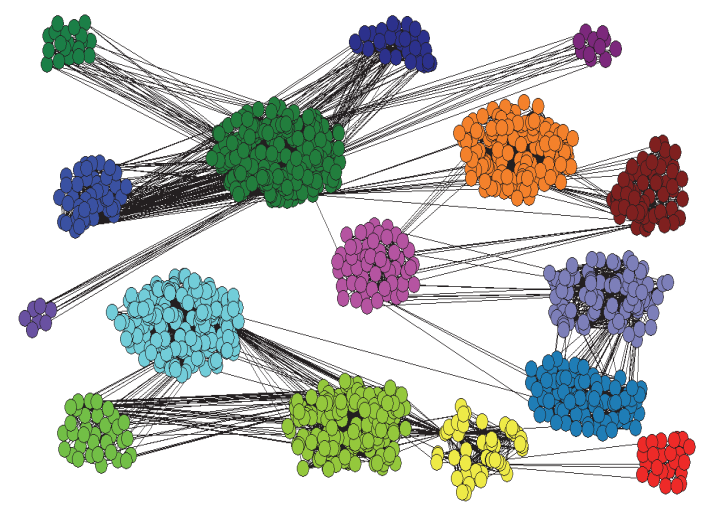

Number of clusters: 16

Modularity: 0.793595

(c) Community detection of CNY against Yen exchange rate network

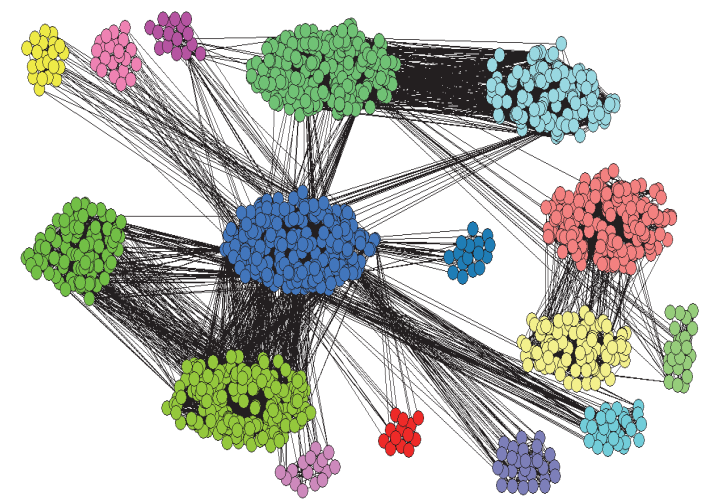

Number of clusters: 16

Modularity: 0.722963

(b) Community detection of CNY against Euro exchange rate network

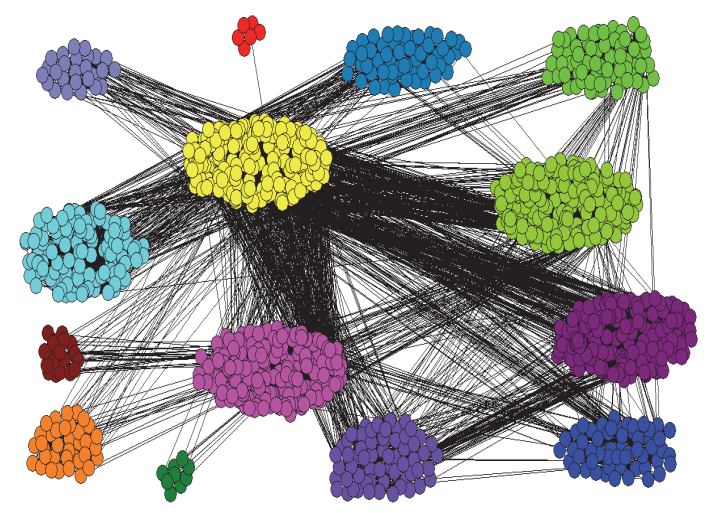

Number of clusters: 14

Modularity: 0.695798

(d) Community detection of CNY against Sterling exchange rate network

FIGURE 6

exchange rate network and also the greatest value of CNY against US dollar exchange rate time series; the maximum value of Euro community 6 is also the fourth-greatest-degree and second-greatest-betweenness-centrality node of whole Euro exchange rate network and also the greatest value of $\mathrm{CNY}$ against Euro exchange rate time series

This is also following the visibility principle: the existence of community containing the most fragments reflects that there is a maximum value connecting those fragments of discontinuous time series corresponding nodes, and thus no other maximum values can divide those less-fluctuating nodes into other communities.

\section{Conclusion}

Although rather large differences of trading goods and capital quantity and structure exist among countries, those CNY against foreign currency exchange rates are correlative and topologically similar, such as average shortest path length, 5 , and average clustering coefficient, 0.7. In addition, from perspectives of hierarchical structures and small-world and scale-free properties, these exchange rate networks are consistent, such as hierarchical exponent, 0.5, and power-law exponent, 1.5 .

On node mixing, four exchange rate networks have some differences. Both US dollar exchange rate network and Sterling exchange rate network are assortative, while both Euro exchange rate network and Yen exchange rate network are disassortative. We propose an explanation that the node mixing formation is determined by the strength comparison of both nodes' interactive force inside group and groups' interactive force among groups. And we use the explanation to reveal time series features.

In order to explore the mechanism of consistency, we utilize the community detection for analysis. We find significant community structures within networks, and additionally those communities consist of large amounts of sequential time series nodes or some fragments of discontinuous time series nodes. Inside community, there tend to be a maximum value, and the population variance of corresponding time series values is significantly smaller than that of whole time series. 


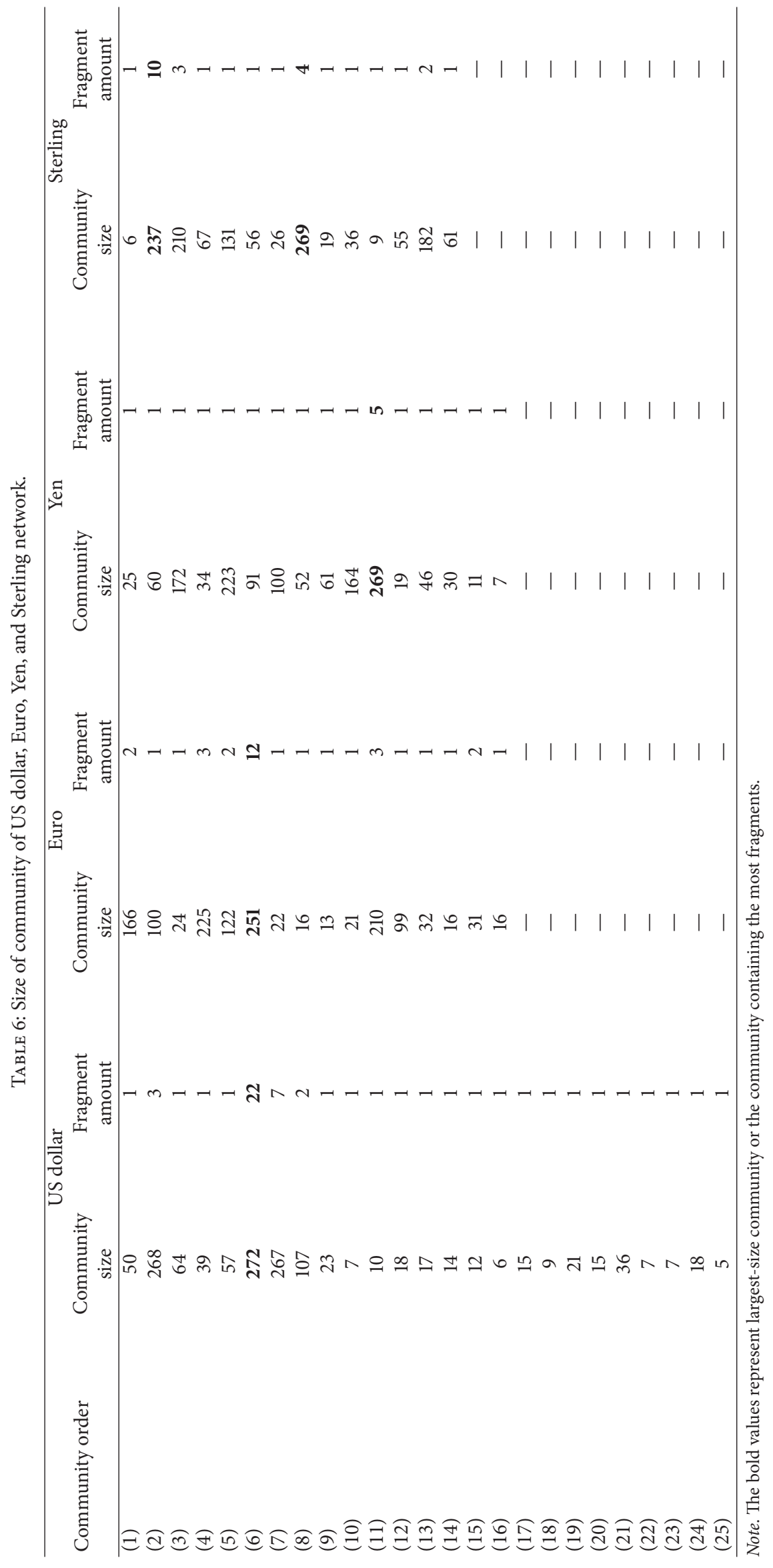


TABLE 7: Statistical results of the community containing the most fragments of US dollar, Euro, Yen, and Sterling exchange rate network.

\begin{tabular}{lcccc}
\hline & US dollar network & US dollar community 6 & Euro network & Euro community 6 \\
\hline Population variance & 726.53 & 163.58 & 3790.75 & 917.38 \\
Maximum value & 685.27 & 681.26 & 1032.38 & 964.13 \\
\hline & Yen network & Yen community 11 & Sterling network & Sterling community 2 \\
\hline Population variance & 0.55 & 0.39 & 2928.44 & 1462.90 \\
Maximum value & 8.37 & 8.17 & 1264.88 & 1264.88 \\
\hline
\end{tabular}

On both time series fluctuating differences and topological structured consistency, we analyze CNY exchange rate network from perspectives of basic topological features and small-world and scale-free properties and explain the fluctuating features as well as their corresponding dynamic effects from topological angle. In addition, we analyze the characteristics of nodes memberships intercommunity and intracommunity. Exchange rate system is a classical complex system with small-world and scale-free properties; however, the time series of exchange rate based on correlative properties are not as simple as long-term correlation.

The visibility graph models we adopt in analyzing exchange rate time series shed light on fluctuation dynamics and characteristics of exchange rate from perspective of nonlinearity. Future work of us may focus on how to incorporate different embedding dimensions and time lags into the construction method of visibility graphs and discuss the visibility graphs in a higher dimension.

\section{Conflicts of Interest}

The authors declare that there are no conflicts of interest regarding the publication of this paper.

\section{Acknowledgments}

This research was supported by the Natural Science Foundation of Guangdong Province of China (Grant 2017A030313396), MOE (Ministry of Education in China) Project of Humanities and Social Sciences (Project no. 17YJAZH109), China State Scholarship Fund (Grant 201706155064), the Fundamental Research Funds for the Central Universities (Grant 2015ZDXM04), and Guangzhou National Innovation-Oriented City Development Research Center (Grant 2017IC02).

\section{References}

[1] D. J. Watts and S. H. Strogatz, "Collective dynamics of 'smallworld' networks," Nature, vol. 393, no. 6684, pp. 440-442, 1998.

[2] A.-L. Barabási and R. Albert, "Emergence of scaling in random networks," American Association for the Advancement of Science: Science, vol. 286, no. 5439, pp. 509-512, 1999.

[3] J. L. Guo, "Bilateral power-law distribution model of supply chain networks," Acta Physica Sinica, vol. 55, no. 8, pp. 39163921, 2006 (Chinese).

[4] Q. Wang and J.-L. Guo, "Human dynamics scaling characteristics for aerial inbound logistics operation," Physica A: Statistical
Mechanics and its Applications, vol. 389, no. 10, pp. 2127-2133, 2010.

[5] W. Souma, Y. Fujiwara, and H. Aoyama, "Complex networks and economics," Physica A: Statistical Mechanics and its Applications, vol. 324, no. 1-2, pp. 396-401, 2003.

[6] S. Ren, X. Cui, and Y. Fan, "Analysis of China's position in international trade based on a complex network perspective," Journal of Beijing Norma University (Natural Science), vol. 1, article 019, 2013.

[7] X. Liu and W. Wang, "Characteristic analysis of complex network for Shanghai stock market," Journal of WUT (Information \& Management Engineering), vol. 34, no. 5, pp. 642-645, 2012.

[8] A. Vazquez, J. G. Oliveira, Z. Dezso, K.-I. Goh, I. Kondor, and A.-L. Barabasi, "Modeling bursts and heavy tails in human dynamics," Physical Review E: Statistical, Nonlinear, and Soft Matter Physics, vol. 73, no. 3, Article ID 036127, 2006.

[9] S. Cheng, "Complexity sciences and management," Bulletin of Chinese Academy of Sciences, vol. 3, pp. 175-183, 1999.

[10] X. Wang, "Challenge of the complexity of market economy," Contemporary Economic Research, vol. 7, pp. 28-32, 2009.

[11] Y. Yang and G. Zong, "A case study of Beijing: Complexity of price fluctuation network in renewable resources industries," Resources \& Industries, vol. 15, no. 001, pp. 148-151, 2013.

[12] Z. Liu, X. Zhuang, and J. Fu, "Complex network characteristics studies on volatility and liquidity of stock market in China," Journal of Northeastern University (Natural Science), vol. 32, no. 11, pp. 1663-1667, 2011.

[13] Y. Yang and H. Yang, "Complex network-based time series analysis," Physica A: Statistical Mechanics and its Applications, vol. 387, no. 5-6, pp. 1381-1386, 2008.

[14] Y. Yang, J. Wang, H. Yang, and J. Mang, "Visibility graph approach to exchange rate series," Physica A: Statistical Mechanics and its Applications, vol. 388, no. 20, pp. 4431-4437, 2009.

[15] L. Lacasa, B. Luque, F. Ballesteros et al., "From time series to complex networks: the visibility graph," Proceedings of the National Acadamy of Sciences of the United States of America, vol. 105, no. 13, pp. 4972-4975, 2008.

[16] W. J. Reed and B. D. Hughes, "From gene families and genera to incomes and internet file sizes: why power laws are so common in nature," Physical Review E: Statistical, Nonlinear, and Soft Matter Physics, vol. 66, no. 6, Article ID 067103, 2002.

[17] P. Bak, C. Tang, and K. Wiesenfeld, "Self-organized criticality: an explanation of the 1/f noise," Physical Review Letters, vol. 59, no. 4, pp. 381-384, 1987.

[18] J. M. Carlson and J. Doyle, "Highly optimized tolerance: robustness and design in complex systems," Physical Review Letters, vol. 84, no. 11, article 2529, 2000.

[19] B. A. Carreras, D. E. Newman, I. Dobson, and A. B. Poole, "Evidence for self-organized criticality in a time series of electric power system blackouts," IEEE Transactions on Circuits and Systems I: Regular Papers, vol. 51, no. 9, pp. 1733-1740, 2004. 
[20] D. Marković and C. Gros, "Power laws and self-organized criticality in theory and nature," Physics Reports, vol. 536, no. 2, pp. 41-74, 2014.

[21] E. Bullmore and O. Sporns, "The economy of brain network organization," Nature Reviews Neuroscience, vol. 13, no. 5, pp. 336-349, 2012.

[22] A. Vespignani, "Modelling dynamical process in complex sociotechnical systems," Nature Physics, vol. 8, no. 1, pp. 32-39, 2012.

[23] A. Clauset, C. R. Shalizi, and M. E. Newman, "Power-law distributions in empirical data," SIAM Review, vol. 51, no. 4, pp. 661-703, 2009.

[24] M. Girvan and M. E. Newman, "Community structure in social and biological networks," Proceedings of the National Acadamy of Sciences of the United States of America, vol. 99, no. 12, pp. 7821-7826, 2002.

[25] M. E. J. Newman and M. Girvan, "Finding and evaluating community structure in networks," Physical Review E: Statistical, Nonlinear, and Soft Matter Physics, vol. 69, no. 2, Article ID 026113, 2004.

[26] V. D. Blondel, J. Guillaume, R. Lambiotte, and E. Lefebvre, "Fast unfolding of communities in large networks," Journal of Statistical Mechanics: Theory and Experiment, vol. 2008, no. 10, Article ID P10008, 2008. 


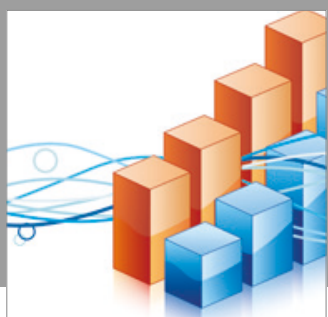

Advances in

Operations Research

vatersals

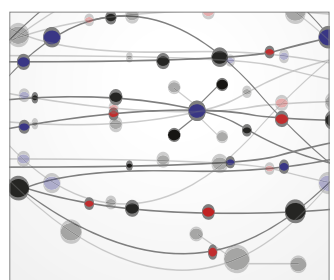

\section{The Scientific} World Journal
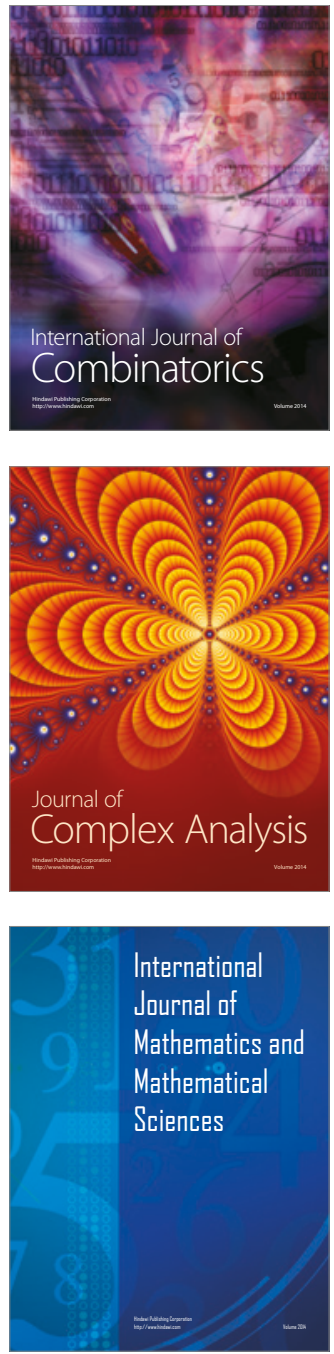
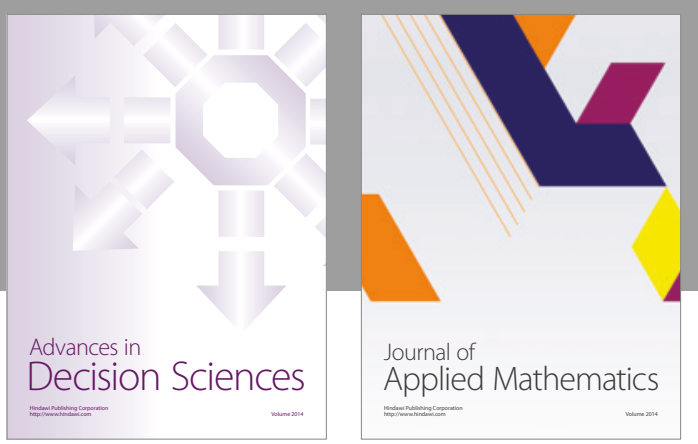

Algebra

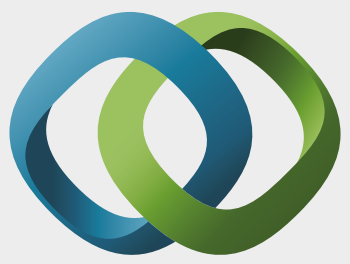

\section{Hindawi}

Submit your manuscripts at

https://www.hindawi.com
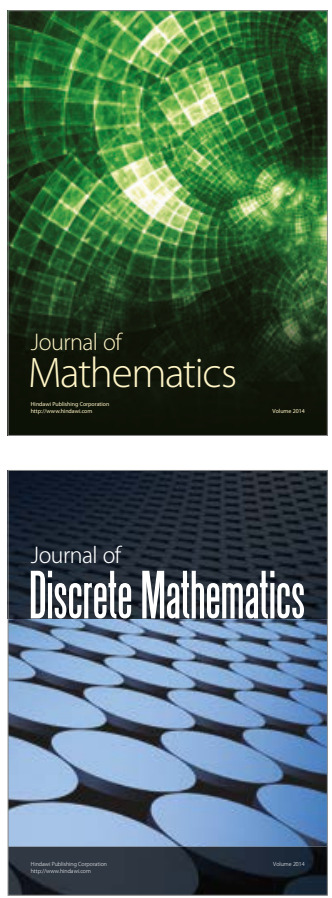

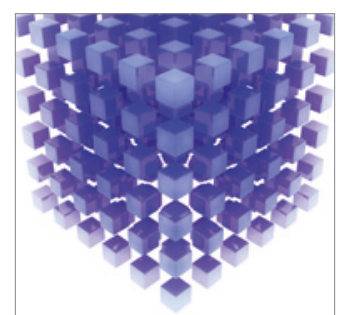

Mathematical Problems in Engineering
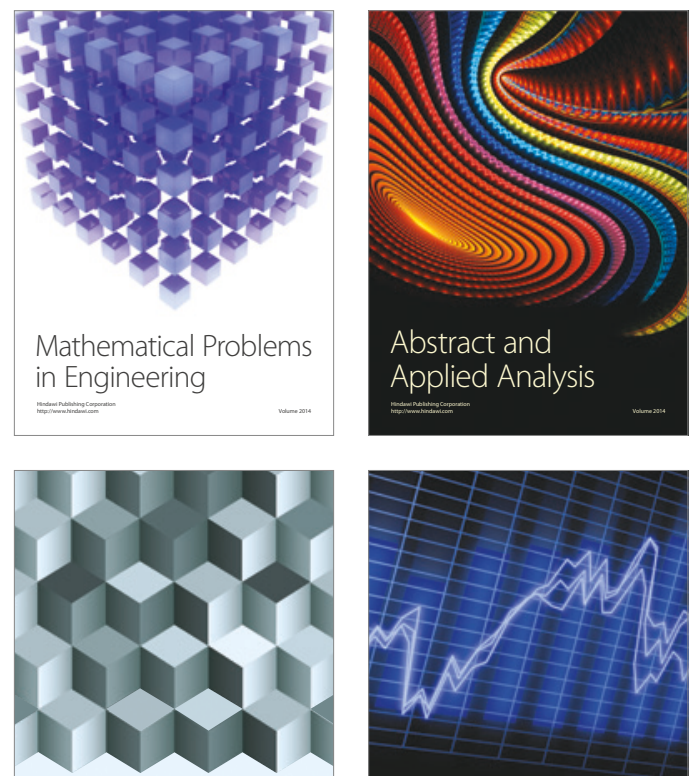

Journal of

Function Spaces

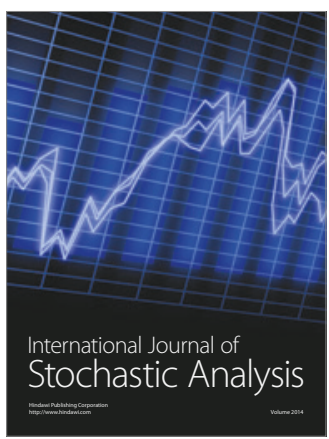

Probability and Statistics
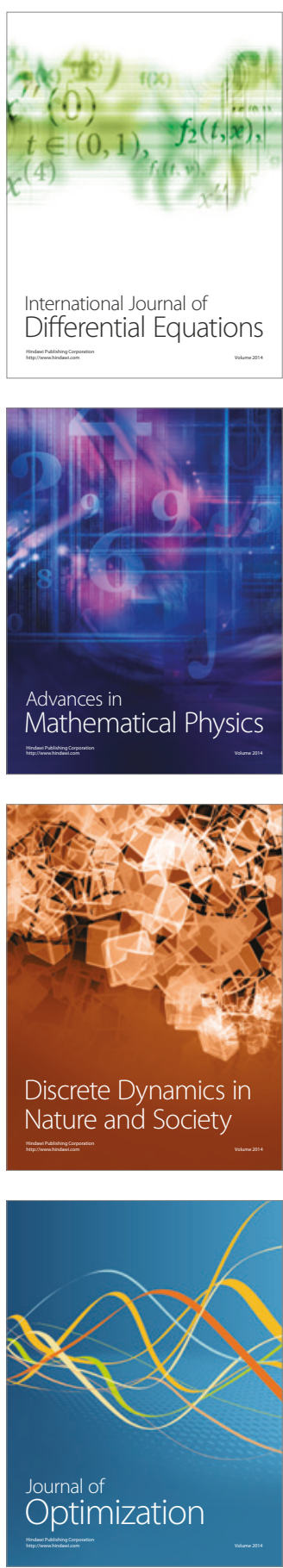